\title{
Lignocellulosic Enzyme Production by Termitomyces spp from Termite Garden
}

\author{
Gomathi V*, M. Esakkiammal S.S. Thilagavathi, A. Ramalakshmi \\ Department of Agricultural Microbiology, Tamil Nadu Agricultural University, India
}

Copyright $\subset 2019$ by authors, all rights reserved. Authors agree that this article remains permanently open access under the terms of the Creative Commons Attribution License 4.0 International License

\begin{abstract}
In nature, microorganisms including fungi are the most efficient cellulose and hemicelluloses degraders through production of lignocellulosic enzymes. Termitomyces spp., a wild edible mushroom is well known for production of lignocellulosic enzymes. Twenty isolates of Termitomyces spp. were isolated at $10^{-4}$ dilution with pour plate method from different locations of termite mound soil of Tamil Nadu. Based on their morphological and cultural characteristics, the isolates were identified as Termitomyces. Growth conditions and cultural characteristics of Termitomyces spp. isolates were studied. The results showed that the isolates grew well in Malt extract agar medium with $\mathrm{pH} 5.5$ when incubated at $27^{\circ} \mathrm{C}$ for 7 days. The isolates were screened for the activity of enzymes e.g., cellulase, amylase, xylanase, polyphenol oxidase and peroxidase activities at seven different incubation periods. Maximum activity of the enzymes was observed on $12^{\text {th }}$ day after inoculation which declined thereafter.
\end{abstract}

Keywords Hemicellulose, Lignocelluloses, Termitomyces spp

\section{Introduction}

About 40-60 million years before the advent of human agriculture, three insect lineages like Termites, Ants and Beetles independently evolved the ability to use fungi for food. Today out of the total more than $2600 \mathrm{sp}$. of termites, 330 are known to form an obligate symbiosis with the specialized fungi e.g., Termitomyces spp. [1]. On the termite comb white spherical yeast like colony fungal structures are commonly observed. These structures of Termitomyces have been described by various authors under different names. Heim [2] proved these structures exclusively as sporodochia which are found on the combs of Macro terminae and represent the anamorph of the genus Termitomyces. In plant cell walls hemicelluloses is a prime biomass reservoir. Agriculture based waste plant products have huge hemicelluloses biomass. Due to this biomass, more efforts have been taken to establish the standard method to degrade this hemicelluloses biomass in last decades [3]. The synergistic activities of termites and different lignocellulose enzymes complex are responsible for degradation of plant cell walls [4]. Makonde et al. 2014[5] described More than 60 Termitomyces taxa but only 18 species, collected from West Africa, are reasonably well known, the rest are either synonyms or badly described and difficult to identify. Commonly associated with woody debris and forest litter, these fungi are able to depolymerize, degrade and fully mineralize all cell wall polymers, including cellulose, hemicelluloses, and the normally rather recalcitrant polymer lignin [6].In addition, termites and other animals have made use of these capacities by utilizing microbial symbionts in the digestion of lignocellulosic food [7]. Different author suggests that Termitomyces spp. produce cellulases and xylanases which degrade cellulose and hemicellulose respectively [8, 9]. In addition, Termitomyces fungi degrade plant lignins to assist cellulose degradation by termites [10,11]. Enzymes like cellulase, amylase and xylanase from Termitomyces clypeatus have been screened and studied for their commercial utility [12]. It is also amenable to large scale cultivation in a bioreactor and gives high productivity of $\mathrm{CDH}$ Termitomyces albuminosus, is a fungus that has a symbiotic relationship with termites, the so-called fungus-growing termites. Fungus-growing termites construct a fungus comb from plant litter, and cultivate Termitomyces albuminosus on this comb as food [2]. The fruiting bodies from inside the tunnels and bore through the very hard layer of inert matter, forcing their way through it with a special umbo [13]. Termitomyces albuminosus partially degrades plant materials, probably including lignin, to help digestion of cellulosic resources by termites [14]. Hence, the present study has been conducted to screen the Termitomyces spp. for lignocellulosic enzyme production.

\section{Materials and Methods}

\subsection{Sampling Site}


A macrofungi Termitomyces spp. and soils from the termite ecosystem were collected from different parts of Tamil Nadu during the monsoon time. The collected samples were taken to the laboratory for isolation purpose under aseptic condition.

\subsection{Termitomyces cultures}

The standard culture used in this study was Termitomyces albuminosus (MTCC 1366) obtained from Institute of Microbial Technology, Chandigarh.

\subsection{Isolation of Termitomyces from termite mound}

Termitomyces were collected from the fresh termite garden, washed with $0.5 \%$ sodium hypo chlorite for 2 minutes and then washed with sterile distilled water for 5 minutes. Finally rinsed with $0.05 \%$ sterile saline. The surface sterilized samples were inoculated in to PDA plates and incubated at room temperature. The plates were observed every day for appearance of fungal colony.

\subsection{Isolation of Termitomyces from fruiting body}

The mushroom tissue was used for isolation of fungi, following the method illustrated by Chang [15]. The mushrooms collected from fungus growing termite mound was washed and disinfected with $75 \%$ alcohol to remove soil debris. The volva was removed and the mushrooms were cut opened by hand longitudinally in halves. Small pieces were aseptically removed from the centre and inoculated on to PDA in which ampicillin (50 ppm) was added as antibacterial agent. Plates were incubated at room temperature $\left(25 \pm 3^{\circ} \mathrm{C}\right)$ in incubated shaker SIF $6000 \mathrm{R} \mathrm{Vol}$ $230 \mathrm{VAC} 50 \mathrm{~Hz}$.

\subsection{Purification}

The different isolates obtained were purified by single hyphal tip method described by Rangasamy et al. [16]. A tip of actively growing mycelium from the individual culture was transferred to solidified malt extract agar (MEA) medium, subsequently to MEA slant and maintained at $4^{\circ} \mathrm{C}$.

\subsection{Factors influencing the growth of Termitomyces}

\subsubsection{Carbon source}

The Termitomyces isolates were grown in malt extract agar media with different carbon sources like glucose, sucrose, starch and malt extract (at the rate of $30 \mathrm{~g} / \mathrm{L}$ ). The plates were incubated for 8 days at room temperature and observed for growth after incubation.

\subsection{2. $\mathrm{pH}$}

Termitomyces isolates were inoculated in Malt extract media having five different $\mathrm{pH}$ ranges e.g., 4.5, 5.5, 6, 7 and 8 and incubated for 8 days at room temperature and observed for their growth.

\subsubsection{Temperature}

Termitomyces isolates were inoculated in Malt extract media and incubated for 8 days at six different temperatures e.g., $0^{\circ} \mathrm{C}, 15^{\circ} \mathrm{C}, 27^{\circ} \mathrm{C}, 37^{\circ} \mathrm{C}, 42^{\circ} \mathrm{C}$ and $50^{\circ} \mathrm{C}$.

\subsection{Characterization of Termitomyces}

Characterization of Termitomyces was done by observing the morphological characters of the mushroom, colony morphology was studied.

\subsection{Macro Morphological Characterization}

Termitomyces was identified by the macro morphological characters described by Tibuhwa et al. [17] and Srivastava et al. [18]. Important characters used for identification were cap size, gills, colour, stipe and pileus.

\subsection{Colony Morphology}

The different Termitomyces isolates were identified by microscopic characterization in compound microscope Magnus MLX under 40x magnifications. Termitomyces isolates were differentiated by their colony characters for example size, shape, colour and consistency [19]. For microscopic identification, the isolates were grown by cover slipculture methods [20] in which individual cultures were transferred to the base of cover slip buried in MEA medium. The cover slip was removed after 8 days of incubation and morphological characters were observed for their mycelial structure, and spore arrangements in microscope. The observed morphology of the isolates was compared with the standard T. albuminosus MTCC 1366 for the presumptive identification of the isolates.

\subsection{Screening of Termitomyces spp. for Biomass Production}

All the isolates were inoculated in MEA $(100 \mathrm{ml})$ containing different carbon source (Carboxy methyl cellulose, starch and xylose) and incubated in room temperature for 12 days. The initial weight of the culture and final weight was recorded and biomass was calculated. The isolates producing more biomass was used for the enzymatic studies.

\subsection{Enzymatic Assays}

The isolates screened based on the biomass production were used for the enzyme activity studies. The cellulolytic activity (Cellulase assay) was determined by colorimetric method [21] using the DNS reagent. The cultures were grown in malt extract broth and the activities were 
measured at 3 days interval till $21^{\text {st }}$ day after inoculation $(0,3$, $6,9,12,15,18$ and 21 days). Two $\mathrm{ml}$ of culture was centrifuged at 13,000 rpm for 5 minutes. From the culture supernatant, $0.1 \mathrm{ml}$ was incubated with $0.9 \mathrm{ml}$ of a CMC solution $(1 \% \mathrm{w} / \mathrm{v})$ in $50 \mathrm{mM}$ sodium phosphate buffer $(\mathrm{pH}$ 7.0) and $0.25 \mathrm{ml}$ distilled water at $40^{\circ} \mathrm{C}$ for 30 minutes. After incubation, the enzyme activity was stopped by adding $1.5 \mathrm{ml}$ DNS-reagent. Then the tubes were placed in boiling water bath at $50^{\circ} \mathrm{C}$ for 15 minutes, cooled down to room temperature. Glucose, used as the standard was prepared at different concentrations 20,40,60,80 and 100 ppm and the assay was carried out as mentioned above. The OD of the sample and standard was immediately measured at $575 \mathrm{~nm}$.

The results were interpreted from the standard graph and expressed in terms of $\mu \mathrm{mol}$ of glucose released $/ \mathrm{min} / \mathrm{mg}$ protein. Chen et al. [22]. $\beta$-Glucosidase activity was estimated at $50^{\circ} \mathrm{C}$ by measuring the release of $p$-nitrophenol from $p$-nitrophenyl- $\beta$-D-glucopyranoside ( $p$ NPG) prepared in citrate buffer [23]. For xylanase the assay mixture contained $0.1 \mathrm{ml}$ enzyme solution, $0.1 \mathrm{ml}$ xylan $(10 \mathrm{mg} / \mathrm{ml}$ on $0.1 \mathrm{~N}$ acetate buffer, $\mathrm{pH} 5.0$ ) and $0.2 \mathrm{ml}$ of the buffer.

The mixture was incubated for $30 \mathrm{~min}$ at $40^{\circ} \mathrm{C}$ and addition of $0.4 \mathrm{ml}$ of alkaline copper reagents stopped the reaction. The mixture was kept for $10 \mathrm{~min}$ in a boiling water bath at $40^{\circ} \mathrm{C}$ and cooled. Then $0.2 \mathrm{ml}$ of arsenomolybdate reagent was added. After 15 minutes, the mixture was centrifuged. Then the supernatant was placed in spectrophotometer at $540 \mathrm{~nm}$. Xylose, used as the standard was prepared at different concentrations 20,40,60,80 and 100 ppm and the assay was carried out.

Amylase assay was carried out by the method of Nelson [23] as modified by Somogyi [24] and also described earlier by Ghosh et al. [25]. The isolates were grown in malt extract media and the assay was carried out by measuring the amount of reducing sugar liberated at three days interval till $21^{\text {st }}$ day after inoculation $(0,3,6,9,12,15$, 18 and 21 days) from the samples. The assay mixture contained $0.02 \mathrm{ml}$ of culture filtrate or enzyme solution, $0 \cdot 2 \mathrm{ml}$ of starch (soluble) suspension or solution $(10 \mathrm{mg} / \mathrm{ml}$ in $0 \cdot 1 \mathrm{M}$ acetate bluffer, $\mathrm{pH} 5 \cdot 0$ ) and $0 \cdot 18 \mathrm{ml}$ of the buffer. Incubation was carried out for $30 \mathrm{~min}$ at $40^{\circ} \mathrm{C}$ and stopped by adding $0.4 \mathrm{ml}$ of alkaline copper reagent. Glucose, used as the standard was prepared at different concentrations $20,40,60,80$ and 100 ppm and the assay was carried out.

Poly phenol oxidase activity was determined by the method described by Zuber and Manibhushanrao [26]. For all the assays the cultures were grown in malt extract broth and the activities were measured at 3 days interval till $21^{\text {st }}$ day after inoculation $(0,3,6,9,12,15,18$ and 21 days) Crude cell extract obtained from the Termitomyces culture grown in malt extract broth was centrifuged at $15,000 \mathrm{rpm}$ for $4^{\circ} \mathrm{C}$ for $15 \mathrm{~min}$ and was used as enzyme source. $2.5 \mathrm{ml}$ of 0.1 M- phosphate buffer ( $\mathrm{pH}$ 6.0) and $0.3 \mathrm{ml}$ of catechol solution $(0.01 \mathrm{M})$ was pipette out in a cuvette. Spectrophotometer was set at $495 \mathrm{~nm}$ at $15,000 \mathrm{rpm}$ for $4^{\circ} \mathrm{C}$ for $15 \mathrm{~min}$. Peroxidase assay was done by the method described by Putter [27]. The assay mixture consisted of 3 $\mathrm{ml}$ of pyrogallol $(0.05 \mathrm{M}), 0.5 \mathrm{ml}$ of the enzyme source and $0.5 \mathrm{ml}$ of $1 \% \mathrm{H}_{2} \mathrm{O}_{2}$. The absorbance was measured in a spectrophotometer at $420 \mathrm{~nm}$ for every 30 s upto 3 minutes.

\section{Results and Discussion}

\section{Isolation of Termitomyces}

Four isolates of Termitomyces spp. were obtained from the soil samples collected from different locations of Tamil $\mathrm{Nadu}$ and sixteen isolates were isolated from the fruiting bodies of Termitomyces collected during the monsoon period. Totally twenty isolates of Termitomyces were purified and designated with isolate numbers (Table 1). 
Table 1. Termitomyces isolates obtained from termite mound samples collected fromdifferent locations.

\begin{tabular}{|c|c|c|}
\hline Place of collection & Isolates & Colony characters on PDA medium \\
\hline \multirow{3}{*}{$\begin{array}{c}\text { Sathyamangalam } 11 \text { o } 30 \text { ' 18.94” N 77o 14' 17.74” E } \\
\text { (Forest ecosystem) }\end{array}$} & $\operatorname{Tm}_{1}$ & White powdery growth \\
\hline & $\mathrm{Tm}_{2}$ & White circular powdery mycelia \\
\hline & $\mathrm{Tm}_{3}$ & White circular mycelia surrounded by slimy layer \\
\hline \multirow{3}{*}{$\begin{array}{l}\text { TNAU (Cultivable land) } 11 \text { o } 077^{\prime} 3.36 " \mathrm{~N} \\
760 \text { o } 59^{\prime} 39.91{ }^{\prime \prime} \mathrm{E}\end{array}$} & $\mathrm{Tm}_{4}$ & White mycelial growth \\
\hline & $\mathrm{Tm}_{5}$ & White powdery growth with brown shade \\
\hline & $\operatorname{Tm}_{6}$ & White mycelial growth with shrinkage \\
\hline \multirow{3}{*}{$\begin{array}{l}\text { TNAU (Cultivable land) } 11 \text { o } 077^{\prime} 3.36 ” \mathrm{~N} \\
76 \text { o } 599^{\prime} 39.91{ }^{\prime \prime} \mathrm{E}\end{array}$} & $\mathrm{Tm}_{7}$ & White powdery growth \\
\hline & $\mathrm{Tm}_{8}$ & White powdery mycelia \\
\hline & $\mathrm{Tm}_{9}$ & Brown powdery mycelia \\
\hline \multirow{7}{*}{$\begin{array}{l}\text { Thalavadi 9o } 20 \text { ' } 59.99 " \mathrm{~N} \\
\text { (Forest ecosystem) 76o 31'59.99”E }\end{array}$} & $\operatorname{Tm}_{10}$ & Brown mycelia surrounded by white powdery growth \\
\hline & $\mathrm{Tm}_{11}$ & White cottony mycelia \\
\hline & $\mathrm{Tm}_{12}$ & White mycelial growth \\
\hline & $\mathrm{Tm}_{13}$ & Brown powdery mycelia \\
\hline & $\mathrm{Tm}_{14}$ & White powdery growth with brown shade \\
\hline & $\mathrm{Tm}_{15}$ & White mycelial growth with shrinkage \\
\hline & $\operatorname{Tm}_{16}$ & White powdery circular colony \\
\hline $\begin{array}{l}\text { Kovai courtallam 10o } 56^{\prime} 20^{\prime \prime} \mathrm{N} \\
\text { (Forest ecosystem) } 76041^{\prime} 20^{\prime \prime} \mathrm{E}\end{array}$ & $\mathrm{T}_{17}$ & White circular powdery growth later turns brown \\
\hline $\begin{array}{l}\text { Pudukottai 10o 37'59.99”N } \\
\text { (Waste land) 18o 07'60.00”E }\end{array}$ & $\mathrm{T}_{18}$ & White circular mycelia later forms spore like structure \\
\hline $\begin{array}{c}\text { Aliyar 10o 28' 26.04”'N } \\
\text { (Cultivable land) 76o 58' 22.08"E }\end{array}$ & $\mathrm{T}_{19}$ & White circular powdery growth \\
\hline $\begin{array}{l}\text { Tirunelveli 8o 43'48.00” } \\
\text { (Cultivable land) 77o 42, } 0.00 ” \mathrm{E}\end{array}$ & $\mathrm{T}_{20}$ & White circular mycelia \\
\hline
\end{tabular}

T1-T16 isolates from fruiting bodies of Termitomyces collected from termite mound; T17-T20 isolates obtained from termite mound soil.

\section{Factors Influencing the Growth of Termitomyces Carbon Source}

All carbon source used in the study supported the growth of Termitomyces spp in Czapek- dox medium whereas starch and malt extract favored the growth of Termitomyces spp compared to other carbon sources. However, malt extract appears to be the preferred carbon source for growth of Termitomyces spp. Glucose and sucrose supported the growth but the incubation period was more (i.e. 12 days) compared to other carbon sources. Growth of the isolates in different carbon sources were recorded and presented (Fig. 1a). 
pH

The media with $\mathrm{pH} 5.5$ favored the growth of the isolates followed by $\mathrm{pH} 4.5$. Least growth was observed in the plates with $\mathrm{pH}$ 8. This experiment showed that $\mathrm{pH} 5.5$ favored the growth of Termitomyces(Fig. 1b).

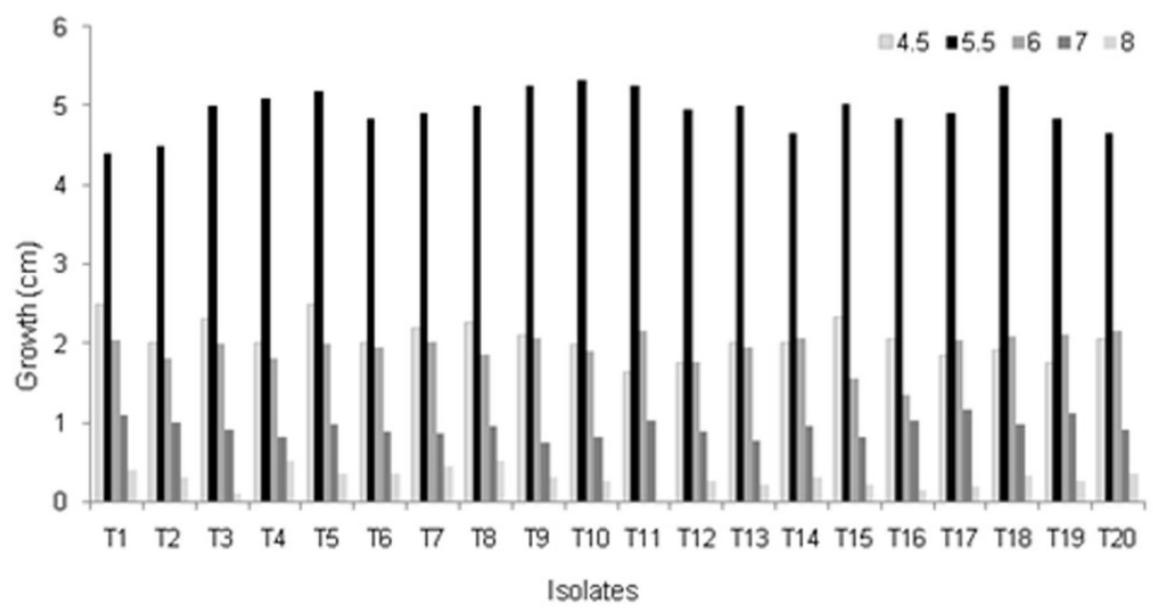

Figure.1b. Effect of $\mathrm{pH}$ on growth of Termitomyces

\section{Temperature}

The growth was better at $27^{\circ} \mathrm{C}$ compared with other temperatures tested. This study showed that Termitomyces spp. preferred the optimum temperature of $27^{\circ} \mathrm{C}$ for its growth (Fig. 1c).

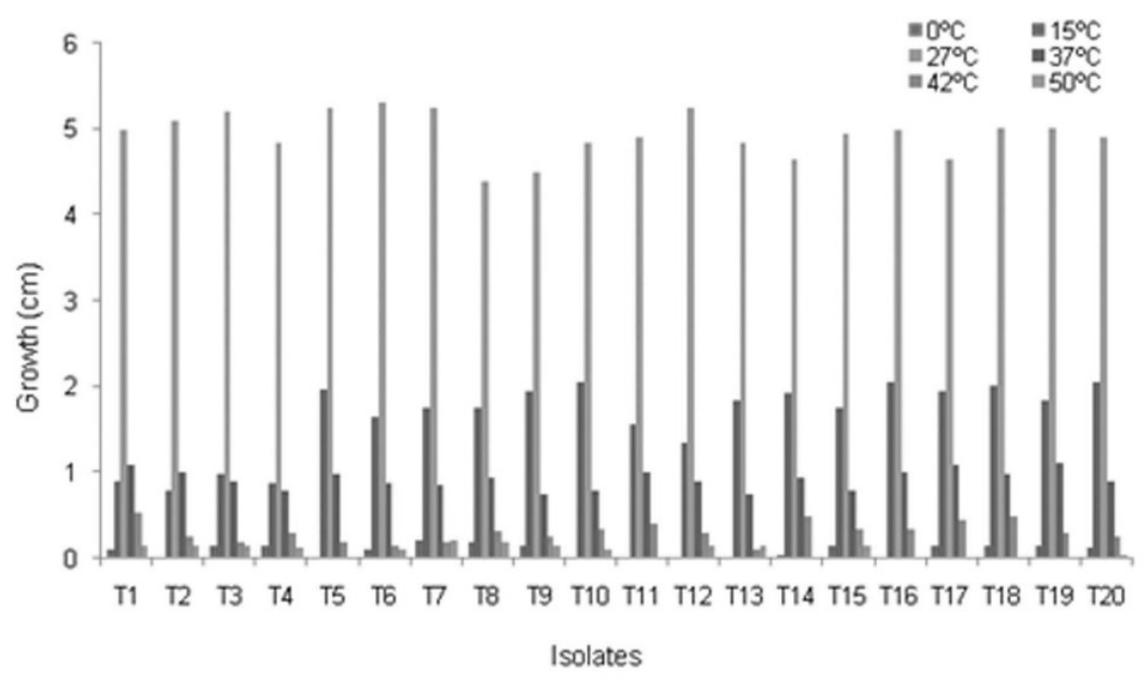

Figure.1c. Effect of temperature on growth of Temitomyces

\section{Characterization of the Isolates}

Macro Morphological Characterization

The colour of stalk was white having long stipe, colour of the cap was varying from dull white to brown.

Colony Morphology

The newest mycelia on the periphery of the culture were usually light and white in colour, while the more aged mycelium toward the centre of the culture were even darker and more differentiated. Mycelia aggregated into elevated forms forming cottony ball-like structures. These balls like structures become dark-brown on the surface of the stroma with age. 


\section{Micro Morphological Characterization}

Under microscopic observation, Termitomyces spp. produced white, turning creamy, soft, hymenophoraltrama regular with thin walled parallel hyphae. Pileal surface had hyphae forming a hypodermal layer below the epicutis consisting of radially parallel, repent, narrow hyphae.

\section{Screening of Termitomyces Spp. Based on Biomass Production}

The experiments resulted in the maximum biomass production of $3.01 \mathrm{~g}$ after 12 days of incubation in the liquid media with $1 \% \mathrm{CMC}$ as carbon source. Only 7 isolates yielded more biomass among the twenty in all the three different carbon sources. Thus the seven isolates screened were used for enzyme activity studies (Fig. 2).

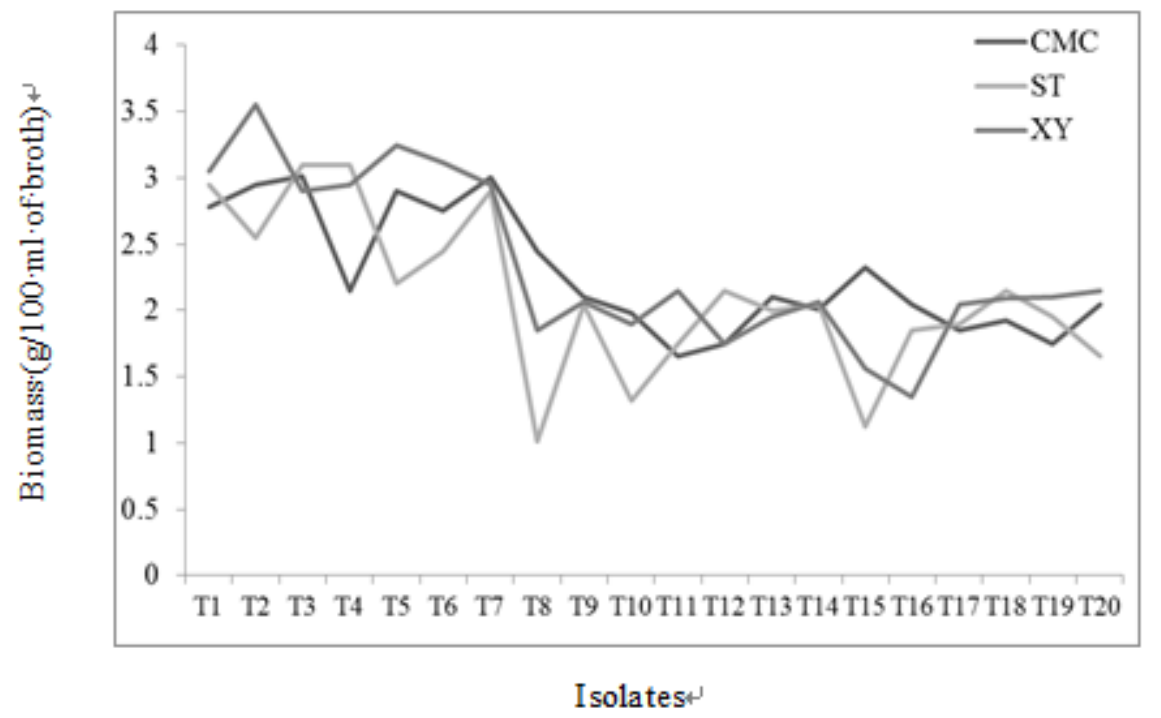

Figure 2. Biomass production of Termitomyces in different carbon sources. (CMC, Carboxy methyl cellulose; ST, Starch; XY, Xylose)

\section{Studies on Enzymes Activity in Termitomyces}

\section{Cellulase Assay}

The cellulase activity of Termitomyces isolates along with standard strain (T. albuminosus MTCC 1366) at different incubation periods was presented in Table 2. All the isolates tested and standard strain MTCC 1366 showed increased cellulase activity up to 12 days of incubation in Czapek-dox broth at room temperature under batch culturing condition and gradually decreased against the incubation time up to 21 days (Table 2). Among the isolates, $\mathrm{Tm}_{3}$ recorded the maximum activity of $10.27 \mu \mathrm{mol}$ of glucose released $/ \mathrm{min} / \mathrm{mg}$ protein at $12^{\text {th }}$ day after inoculation followed by the standard strain (T. albuminosus MTCC 1366). Among the isolates, $\mathrm{Tm}_{5}$ had least activity on $12^{\text {th }}$ day $(9.7 \mu$ mol of glucose released/min/mg protein).

Table 2. Cellulase activity of different Termitomyces isolates

\begin{tabular}{ccccccccc}
\hline \multicolumn{7}{c}{ Cellulase activity $(\boldsymbol{\mu}$ mol of glucose released/min/mg protein) } \\
\hline Isolates & $\mathbf{0}^{\text {th }} \mathbf{d a y}$ & $\mathbf{3}^{\text {rd }} \mathbf{d a y}$ & $\mathbf{6}^{\text {th }}$ day & $\mathbf{9}^{\text {th }}$ day & $\mathbf{1 2}^{\text {th }} \mathbf{d a y}$ & $\mathbf{1 5}^{\text {th }} \mathbf{d a y}$ & $\mathbf{1 8}^{\text {th }} \mathbf{d a y}$ & $\mathbf{2 1}^{\text {st }} \mathbf{d a y}$ \\
\hline $\mathrm{T} 1$ & $6.43 \pm 0.72 \mathrm{c}$ & $6.93 \pm 0.54 \mathrm{c}$ & $7.27 \pm 0.31 \mathrm{c}$ & $7.90 \pm 0.41 \mathrm{~b}$ & $10.07 \pm 0.43 \mathrm{a}$ & $8.17 \pm 0.72 \mathrm{~b}$ & $7.57 \pm 0.31 \mathrm{c}$ & $6.10 \pm 0.66 \mathrm{c}$ \\
$\mathrm{T} 2$ & $5.50 \pm 0.4 \mathrm{c}$ & $6.27 \pm 0.31 \mathrm{c}$ & $6.97 \pm 0.12 \mathrm{c}$ & $7.97 \pm 0.20 \mathrm{~b}$ & $9.87 \pm 0.26 \mathrm{a}$ & $9.27 \pm 0.27 \mathrm{a}$ & $7.87 \pm 0.29 \mathrm{~b}$ & $7.13 \pm 0.18 \mathrm{~b}$ \\
$\mathrm{~T} 3$ & $6.03 \pm 0.44 \mathrm{c}$ & $6.50 \pm 0.29 \mathrm{c}$ & $7.20 \pm 0 \mathrm{c}$ & $7.90 \pm 0.35 \mathrm{~b}$ & $10.27 \pm 0.26 \mathrm{a}$ & $8.87 \pm 0.40 \mathrm{~b}$ & $8.37 \pm 0.33 \mathrm{~b}$ & $6.80 \pm 0.86 \mathrm{c}$ \\
$\mathrm{T} 4$ & $5.83 \pm 0.83 \mathrm{~d}$ & $6.73 \pm 0.63 \mathrm{~d}$ & $7.60 \pm 0.35 \mathrm{c}$ & $8.43 \pm 0.14 \mathrm{~b}$ & $9.93 \pm 0.34 \mathrm{a}$ & $8.67 \pm 0.68 \mathrm{~b}$ & $7.43 \pm 0.31 \mathrm{c}$ & $9.70 \pm 0.3 \mathrm{a}$ \\
$\mathrm{T} 5$ & $5.83 \pm 0.44 \mathrm{~d}$ & $6.53 \pm 0.26 \mathrm{~d}$ & $7.17 \pm 0.13 \mathrm{c}$ & $8.13 \pm 0.19 \mathrm{~b}$ & $9.70 \pm 0.11 \mathrm{a}$ & $8.93 \pm 0.15 \mathrm{~b}$ & $8.20 \pm 0.15 \mathrm{~b}$ & $7.50 \pm 0.15 \mathrm{c}$ \\
$\mathrm{T} 6$ & $6.43 \pm 0.55 \mathrm{~d}$ & $7.10 \pm 0.63 \mathrm{c}$ & $7.47 \pm 0.29 \mathrm{c}$ & $7.87 \pm 0.35 \mathrm{c}$ & $10.20 \pm 0.4 \mathrm{a}$ & $8.47 \pm 0.68 \mathrm{~b}$ & $7.50 \pm 0.57 \mathrm{c}$ & $6.20 \pm 0.75 \mathrm{~d}$ \\
$\mathrm{~T} 7$ & $5.43 \pm 0.29 \mathrm{c}$ & $6.50 \pm 0.25 \mathrm{c}$ & $7.50 \pm 0 \mathrm{~b}$ & $8.63 \pm 0.13 \mathrm{~b}$ & $10.10 \pm 0.15 \mathrm{a}$ & $9.00 \pm 0.21 \mathrm{a}$ & $7.63 \pm 0.31 \mathrm{~b}$ & $6.90 \pm 0.2 \mathrm{c}$ \\
$\mathrm{Ta}$ & $5.77 \pm 0.28 \mathrm{~d}$ & $6.47 \pm 0.21 \mathrm{~d}$ & $7.37 \pm 0.13 \mathrm{c}$ & $8.27 \pm 0.15 \mathrm{~b}$ & $10.03 \pm 0.33 \mathrm{a}$ & $9.30 \pm 0.33 \mathrm{a}$ & $8.20 \pm 0.1 \mathrm{~b}$ & $7.57 \pm 0.28 \mathrm{c}$ \\
\hline
\end{tabular}

Ta-Termitomyces albuminosus (MTCC 1366). Values are mean \pm SE

Means in a columan followed by a same letter(s) are not significantly $(p \leq 0.05)$ different according to DMRT. 


\section{Xylanase Assay}

All the seven isolates obtained from fruiting body and the standard strain (T. albuminosus MTCC 1366) exhibited xylanse activity (Table 3 ). The isolate $\mathrm{Tm}_{4}$ showed maximum activity of $9.87 \mu \mathrm{mol}$ of xylose released/ $\mathrm{min} / \mathrm{mg}$ protein followed by the isolates $\mathrm{Tm}_{6}$ and $\mathrm{Tm}_{7}$ on $12^{\text {th }}$ day of incubation, which was however on par with the standard culture $(T$. albuminosus MTCC 1366) recording the activity of $9.77 \mu \mathrm{mol}$ of xylose liberated $/ \mathrm{min} / \mathrm{mg}$ protein on $12^{\text {th }}$ day of incubation. In all the isolates, the maximum xylanase activity was recorded on $12^{\text {th }}$ day and was decreased gradually over incubation.

Table 3. Xylanase activity of different Termitomyces isolates.

\begin{tabular}{ccccccccc}
\hline \multicolumn{7}{c}{ Xylanase activity $(\boldsymbol{\mu}$ mol of xylose released/min/mg protein) } \\
\hline Isolates & $\mathbf{0}^{\text {th }}$ day & $\mathbf{3}^{\text {rd }} \mathbf{d a y}$ & $\mathbf{6}^{\text {th }}$ day & $\mathbf{9}^{\text {th }}$ day & $\mathbf{1 2}^{\text {th }} \mathbf{d a y}$ & $\mathbf{1 5}^{\text {th }} \mathbf{d a y}$ & $\mathbf{1 8}^{\text {th }} \mathbf{d a y}$ & $\mathbf{2 1}^{\text {st }} \mathbf{d a y}$ \\
\hline $\mathrm{T} 1$ & $6.87 \pm 0.46 \mathrm{~d}$ & $7.40 \pm 0.2 \mathrm{c}$ & $7.77 \pm 0.23 \mathrm{c}$ & $8.80 \pm 0.1 \mathrm{~b}$ & $9.67 \pm 0.18 \mathrm{a}$ & $8.77 \pm 0.23 \mathrm{~b}$ & $8.23 \pm 0.14 \mathrm{~b}$ & $7.13 \pm 0.08 \mathrm{c}$ \\
$\mathrm{T} 2$ & $6.90 \pm 0.25 \mathrm{c}$ & $7.37 \pm 0.2 \mathrm{~b}$ & $7.63 \pm 0.42 \mathrm{~b}$ & $8.43 \pm 0.56 \mathrm{a}$ & $9.73 \pm 0.17 \mathrm{a}$ & $8.97 \pm 0.03 \mathrm{a}$ & $7.86 \pm 0.43 \mathrm{~b}$ & $6.47 \pm 0.51 \mathrm{c}$ \\
$\mathrm{T} 3$ & $6.93 \pm 0.13 \mathrm{~d}$ & $7.20 \pm 0.15 \mathrm{c}$ & $7.90 \pm 0.15 \mathrm{c}$ & $8.37 \pm 0.23 \mathrm{~b}$ & $9.60 \pm 0.1 \mathrm{a}$ & $9.10 \pm 0.43 \mathrm{a}$ & $7.60 \pm 0.35 \mathrm{c}$ & $6.90 \pm 0.05 \mathrm{~d}$ \\
$\mathrm{~T} 4$ & $6.90 \pm 0.37 \mathrm{c}$ & $7.53 \pm 0.24 \mathrm{~b}$ & $7.87 \pm 0.24 \mathrm{~b}$ & $8.60 \pm 0.20 \mathrm{a}$ & $9.87 \pm 0.08 \mathrm{a}$ & $8.90 \pm 0 \mathrm{a}$ & $8.00 \pm 0.05 \mathrm{~b}$ & $7.06 \pm 0.08 \mathrm{~b}$ \\
$\mathrm{~T} 5$ & $6.97 \pm 0.17 \mathrm{~d}$ & $7.40 \pm 0.3 \mathrm{c}$ & $7.77 \pm 0.46 \mathrm{c}$ & $8.47 \pm 0.42 \mathrm{~b}$ & $9.70 \pm 0.11 \mathrm{a}$ & $9.03 \pm 0.33 \mathrm{a}$ & $7.36 \pm 0.31 \mathrm{c}$ & $6.67 \pm 0.37 \mathrm{~d}$ \\
$\mathrm{~T} 6$ & $6.77 \pm 0.35 \mathrm{c}$ & $7.37 \pm 0.27 \mathrm{~b}$ & $7.83 \pm 0.29$ & $8.93 \pm 0.17 \mathrm{a}$ & $9.77 \pm 0.16 \mathrm{a}$ & $8.66 \pm 0.18 \mathrm{a}$ & $7.80 \pm 0.15 \mathrm{~b}$ & $6.90 \pm 0.26 \mathrm{c}$ \\
$\mathrm{T} 7$ & $7.20 \pm 0.28 \mathrm{~b}$ & $7.50 \pm 0.26 \mathrm{~b}$ & $7.67 \pm 0.33 \mathrm{~b}$ & $8.23 \pm 0.12 \mathrm{a}$ & $9.77 \pm 0.03 \mathrm{a}$ & $8.60 \pm 0.15 \mathrm{a}$ & $7.83 \pm 0.21 \mathrm{~b}$ & $6.93 \pm 0.03 \mathrm{c}$ \\
$\mathrm{Ta}$ & $6.90 \pm 0.20 \mathrm{c}$ & $7.67 \pm 0.24 \mathrm{~b}$ & $8.10 \pm 0.15 \mathrm{~b}$ & $8.73 \pm 0.23 \mathrm{a}$ & $9.77 \pm 0.14 \mathrm{a}$ & $8.97 \pm 0.26 \mathrm{a}$ & $7.17 \pm 0.12 \mathrm{~b}$ & $6.80 \pm 0.23 \mathrm{c}$ \\
\hline
\end{tabular}

$\mathrm{Ta}-$ Termitomyces albuminosus (MTCC 1366). Values are mean $\pm \mathrm{SE}$.

Means in a columan followed by a same letter(s) are not significantly $(p \leq 0.05)$ different according to DMRT

Amylase Assay

All the isolates exhibited higher activity on $12^{\text {th }}$ day of incubation and results are presented in Table 3 . Isolate $\mathrm{Tm}_{6}$ showed maximum amylase activity of $14.5 \mu \mathrm{mol}$ of glucose released $/ \mathrm{min} / \mathrm{mg}$ protein on $12^{\text {th }}$ day of incubation, followed by $\mathrm{T}_{3}(13.77 \mu \mathrm{mol}$ of glucose released/ $\mathrm{min} / \mathrm{mg}$ protein $)$. The standard culture showed least activity of $13 \mu \mathrm{mol}$ of glucose released/ $\mathrm{min} / \mathrm{mg}$ protein on $12^{\text {th }}$ day of incubation (Table 4 ).

Table 4. Amylase activity of different Termitomyces isolates.

\begin{tabular}{|c|c|c|c|c|c|c|c|c|}
\hline \multicolumn{9}{|c|}{ Amylase activity ( $\mu$ mol of glucose released/min/mg protein) } \\
\hline Isolates & 0th day & 3rd day & 6th day & 9th day & 12th day & 15th day & 18th day & 21st day \\
\hline $\mathrm{T} 1$ & $8.93 \pm 1.0 \mathrm{c}$ & $9.83 \pm 0.89 \mathrm{c}$ & $10.83 \pm 1.4 \mathrm{~b}$ & $12.13 \pm 1.34 \mathrm{a}$ & $\begin{array}{c}13.73 \\
\pm 1.62 \mathrm{a}\end{array}$ & $12.47 \pm 2.13 \mathrm{a}$ & $\begin{array}{c}10.37 \\
\pm 1.25 \mathrm{~b}\end{array}$ & $8.70 \pm 0.86 \mathrm{c}$ \\
\hline $\mathrm{T} 2$ & $6.73 \pm 0.31 \mathrm{e}$ & $7.97 \pm 0.43 \mathrm{~d}$ & $8.93 \pm 0.66 \mathrm{c}$ & $9.87 \pm 0.73 b$ & $\begin{array}{c}11.73 \\
\pm 1.02 \mathrm{a}\end{array}$ & $9.77 \pm 1.47 \mathrm{~b}$ & $\begin{array}{c}9.00 \\
\pm 1.04 \mathrm{~b}\end{array}$ & $8.27 \pm 0.99 \mathrm{c}$ \\
\hline $\mathrm{T} 3$ & $9.67 \pm 0.84 \mathrm{e}$ & $10.27 \pm 0.71 \mathrm{~d}$ & $11.20 \pm 0.98 \mathrm{c}$ & $12.00 \pm 1.04 \mathrm{~b}$ & $\begin{array}{c}13.77 \\
\pm 0.72 \mathrm{a}\end{array}$ & $12.83 \pm 0.83 b$ & $\begin{array}{c}10.70 \\
\pm 0.15 \mathrm{~d}\end{array}$ & $8.90 \pm 0.95 \mathrm{e}$ \\
\hline $\mathrm{T} 4$ & $7.43 \pm 0.88 \mathrm{e}$ & $8.57 \pm 0.99 \mathrm{~d}$ & $9.17 \pm 0.98 \mathrm{c}$ & $10.67 \pm 1.46 b$ & $11.73 \pm 1.8 \mathrm{a}$ & $10.002 .29 b$ & $\begin{array}{c}8.90 \\
\pm 1.07 \mathrm{~d}\end{array}$ & $7.80 \pm 0.90 \mathrm{e}$ \\
\hline T5 & $8.50 \pm 1.01 \mathrm{e}$ & $9.40 \pm 0.70 \mathrm{~d}$ & $10.17 \pm 0.66 \mathrm{c}$ & $11.13 \pm 0.46 b$ & $\begin{array}{c}13.10 \\
\pm 0.25 \mathrm{a}\end{array}$ & $11.90 \pm 0.50 \mathrm{~b}$ & $\begin{array}{c}10.33 \\
\pm 0.46 \mathrm{c}\end{array}$ & $9.27 \pm 0.31 \mathrm{~d}$ \\
\hline T6 & $9.27 \pm 0.76 \mathrm{e}$ & $10.37 \pm 0.72 \mathrm{~d}$ & $11.47 \pm 1.01 \mathrm{~d}$ & $12.60 \pm 1.0 \mathrm{c}$ & $\begin{array}{c}14.50 \\
\pm 0.85 \mathrm{a}\end{array}$ & $13.50 \pm 0.8 \mathrm{~b}$ & $\begin{array}{c}11.03 \\
\pm 0.63 \mathrm{~d}\end{array}$ & $8.70 \pm 0.62 \mathrm{e}$ \\
\hline $\mathrm{T} 7$ & $6.8 \pm 0.40 \mathrm{e}$ & $8.03 \pm 0.55 \mathrm{~d}$ & $8.93 \pm 0.71 \mathrm{~d}$ & $10.06 \pm 0.69 b$ & $\begin{array}{c}11.33 \\
\pm 1.23 \mathrm{a}\end{array}$ & $9.17 \pm 1.58 \mathrm{c}$ & $\begin{array}{c}8.33 \\
\pm 1.15 \mathrm{~d}\end{array}$ & $7.53 \pm 0.95 \mathrm{e}$ \\
\hline $\mathrm{Ta}$ & $8.87 \pm 0.77 \mathrm{e}$ & $9.67 \pm 0.63 \mathrm{~d}$ & $9.67 \pm 0.71 \mathrm{~d}$ & $11.06 \pm 0.47 \mathrm{~b}$ & $13.00 \pm 0.2 \mathrm{a}$ & $11.6 \pm 0.60 \mathrm{~b}$ & $\begin{array}{c}10.23 \\
\pm 0.37 \mathrm{c}\end{array}$ & $8.57 \pm 0.67 \mathrm{~d}$ \\
\hline
\end{tabular}

$\mathrm{Ta}-$ Termitomyces albuminosus (MTCC 1366). Values are mean \pm SE.

Means in a column followed by a same letter (s) are not significantly $(p \leq 0.05)$ different according to DMRT.

\section{Polyphenoloxidase Assay}

The isolate $\mathrm{Tm}_{7}$ exhibited the maximum activity of $3.80 \mathrm{OD}$ change/min $/ \mathrm{mg}$ protein on $12^{\text {th }}$ day of incubation followed by $\operatorname{Tm}_{2}$ (2.93 OD change/min/mg protein) (Table 5). The standard culture exhibited mean enzyme activity of 2.91 OD change $/ \mathrm{min} / \mathrm{mg}$ protein. Least activity was observed in the isolate $\mathrm{Tm}_{6}(2.73 \mathrm{OD}$ change $/ \mathrm{min} / \mathrm{mg}$ protein $)$ on $12^{\text {th }}$ day of incubation. 
Table. 5. Poly phenol oxidase activity of different Termitomyces isolates

\begin{tabular}{|c|c|c|c|c|c|c|c|c|}
\hline \multicolumn{9}{|c|}{ Poly phenol oxidase activity (OD change/min/mg protein) } \\
\hline Isolates & $0^{\text {th }}$ day & $3^{\text {rd }}$ day & $6^{\text {th }}$ day & $9^{\text {th }}$ day & $12^{\text {th }}$ day & $15^{\text {th }}$ day & $18^{\text {th }}$ day & $21^{\text {st }}$ day \\
\hline $\mathrm{T} 1$ & $1.06 \pm 0.08 \mathrm{~b}$ & $1.36 \pm 0.13 b$ & $2.20 \pm 0.55 \mathrm{a}$ & $2.17 \pm 0.66 \mathrm{a}$ & $\begin{array}{c}2.87 \\
\pm 0.89 \mathrm{a}\end{array}$ & $2.20 \pm 0.81 \mathrm{a}$ & $1.17 \pm 0.20 \mathrm{~b}$ & $0.90 \pm 0 \mathrm{~b}$ \\
\hline $\mathrm{T} 2$ & $1.05 \pm 0.03 b$ & $1.87 \pm 0.08 b$ & $2.40 \pm 0.17 \mathrm{a}$ & $2.60 \pm 0.45 \mathrm{a}$ & $\begin{array}{c}2.93 \\
\pm 0.48 \mathrm{a}\end{array}$ & $2.67 \pm 0.29 \mathrm{a}$ & $1.93 \pm 052 b$ & $1.20 \pm 0.4 \mathrm{~b}$ \\
\hline $\mathrm{T} 3$ & $1.57 \pm 0.32 \mathrm{~b}$ & $1.93 \pm 0.29 b$ & $2.57 \pm 0.34 \mathrm{a}$ & $2.70 \pm 0.52 \mathrm{a}$ & $\begin{array}{c}2.90 \\
\pm 0.72 \mathrm{a}\end{array}$ & $2.23 \pm 0.63 a$ & $1.06 \pm 0.12 b$ & $0.90 \pm 0.1 \mathrm{c}$ \\
\hline $\mathrm{T} 4$ & $1.30 \pm 0.26 \mathrm{c}$ & $1.6 \pm 0.25 b$ & $2.03 \pm 0.24 \mathrm{a}$ & $2.08 \pm 0.54 \mathrm{a}$ & $\begin{array}{c}2.83 \\
\pm 0.73 \mathrm{a}\end{array}$ & $1.80 \pm 0.55 b$ & $1.33 \pm 0.34 \mathrm{c}$ & $0.80 \pm 0.05 c$ \\
\hline T5 & $1.07 \pm 0.1 b$ & $1.9 \pm 0.1 b$ & $2.37 \pm 0.23 \mathrm{a}$ & $2.63 \pm 0.47 \mathrm{a}$ & $\begin{array}{c}2.87 \\
\pm 0.51 \mathrm{a}\end{array}$ & $2.40 \pm 0.36 \mathrm{a}$ & $1.43 \pm 0.53 b$ & $1.10 \pm 0.40 \mathrm{~b}$ \\
\hline T6 & $1.43 \pm 0.33 b$ & $1.87 \pm 0.27 b$ & $2.37 \pm 0.37 \mathrm{a}$ & $2.00 \pm 0.58 \mathrm{a}$ & $\begin{array}{c}2.73 \\
\pm 0.88 \mathrm{a}\end{array}$ & $1.83 \pm 0.69 b$ & $0.97 \pm 0.12 \mathrm{c}$ & $0.83 \pm 0.03 \mathrm{c}$ \\
\hline $\mathrm{T} 7$ & $1.50 \pm 0.25 \mathrm{c}$ & $1.97 \pm 0.24 \mathrm{c}$ & $2.43 \pm 0.29 \mathrm{~b}$ & $2.87 \pm 0.23 b$ & $\begin{array}{c}3.80 \\
\pm 0.15 \mathrm{a}\end{array}$ & $2.50 \pm 0.36 \mathrm{~b}$ & $1.70 \pm 0.35 \mathrm{c}$ & $1.00 \pm 0.26 \mathrm{~d}$ \\
\hline Ta & $1.80 \pm 0.11 \mathrm{~b}$ & $2.03 \pm 0.13 b$ & $2.43 \pm 0.12 \mathrm{a}$ & $2.10 \pm 0.50 \mathrm{a}$ & $\begin{array}{c}2.91 \\
\pm 0.13 \mathrm{a}\end{array}$ & $1.97 \pm 0.21 \mathrm{~b}$ & $1.20 \pm 0.15 \mathrm{c}$ & $0.83 \pm 0.03 \mathrm{c}$ \\
\hline
\end{tabular}

Ta - Termitomyces albuminosus (MTCC 1366). Values are mean \pm SE.

Means in a column followed by a same letter (s) are not significantly $(p \leq 0.05)$ different according to DMRT.

Peroxidase Assay

The isolate $\mathrm{Tm}_{4}$ exhibited more peroxidaseactivity (3.87 OD change/min $/ \mathrm{mg}$ protein) followed by the isolate $\mathrm{Tm} \mathrm{m}_{1}(3.70$ OD change $/ \mathrm{min} / \mathrm{mg}$ protein) on $12^{\text {th }}$ day of incubation (Table 6 ). The standard culture exhibited mean enzyme activity of $3.52 \mathrm{OD}$ change $/ \mathrm{min} / \mathrm{mg}$ protein. Least activity was exhibited by the isolate $\operatorname{Tm}_{5}(2.27 \mathrm{OD}$ change/min $/ \mathrm{mg} \mathrm{protein})$.

Table 6. Peroxidase activity of different Termitomyces isolates

\begin{tabular}{|c|c|c|c|c|c|c|c|c|}
\hline \multirow[b]{2}{*}{ Isolates } & \multicolumn{8}{|c|}{ Peroxidase activity (OD change/min/mg protein) } \\
\hline & $0^{\text {th }}$ day & $3^{\text {rd }}$ day & $6^{\text {th }}$ day & $9^{\text {th }}$ day & $12^{\text {th }}$ day & $15^{\text {th }}$ day & $18^{\text {th }}$ day & $21^{\text {st }}$ day \\
\hline $\mathrm{T} 1$ & $\begin{array}{c}1.37 \\
\pm 0.13 \mathrm{c}\end{array}$ & $1.7 \pm 0.20 \mathrm{c}$ & $1.77 \pm 0.12 \mathrm{c}$ & $1.37 \pm 0.3 \mathrm{c}$ & $\begin{array}{c}3.70 \\
\pm 0.35 \mathrm{a}\end{array}$ & $2.27 \pm 0.52 b$ & $1.50 \pm 0.25 \mathrm{c}$ & $0.93 \pm 0.03 \mathrm{~d}$ \\
\hline $\mathrm{T} 2$ & $\begin{array}{c}1.70 \\
\pm 0.25 \mathrm{~b}\end{array}$ & $1.87 \pm 0.27 \mathrm{~b}$ & $2.73 \pm 0.31 \mathrm{a}$ & $2.40 \pm 0.50 \mathrm{a}$ & $\begin{array}{c}2.63 \\
\pm 0.47 \mathrm{a}\end{array}$ & $1.73 \pm 0.14 \mathrm{~b}$ & $1.230 .17 \mathrm{~b}$ & $0.93 \pm 0.08 \mathrm{c}$ \\
\hline $\mathrm{T} 3$ & $\begin{array}{c}1.83 \\
\pm 0.23 \mathrm{c}\end{array}$ & $1.93 \pm 0.23 \mathrm{c}$ & $2.43 \pm 0.17 b$ & $1.93 \pm 0.63$ & $\begin{array}{c}3.07 \\
\pm 0.31 \mathrm{a}\end{array}$ & $2.33 \pm 0.69 b$ & $1.10 \pm 0.05 \mathrm{c}$ & $0.83 \pm 0.03 \mathrm{~d}$ \\
\hline $\mathrm{T} 4$ & $\begin{array}{c}1.47 \\
\pm 0.24 \mathrm{c}\end{array}$ & $1.87 \pm 0.18 \mathrm{c}$ & $2.13 \pm 0.33 b$ & $1.87 \pm 0.23 \mathrm{c}$ & $\begin{array}{c}3.87 \\
\pm 0.06 \mathrm{a}\end{array}$ & $2.43 \pm 0.29 b$ & $1.70 \pm 0.15 \mathrm{c}$ & $0.90 \pm 0 \mathrm{~d}$ \\
\hline T5 & $\begin{array}{c}1.77 \pm 0.3 \\
3 \mathrm{~b}\end{array}$ & $2.2 \pm 0.20 \mathrm{a}$ & $2.5 \pm 0.37 \mathrm{a}$ & $2.43 \pm 0.53 \mathrm{a}$ & $\begin{array}{c}2.27 \\
\pm 0.13 \mathrm{a}\end{array}$ & $1.67 \pm 0.14 b$ & $0.97 \pm 0.12 \mathrm{c}$ & $0.83 \pm 0.12 \mathrm{c}$ \\
\hline T6 & $\begin{array}{c}1.67 \\
\pm 0.08 \mathrm{c}\end{array}$ & $2.03 \pm 0.08 \mathrm{~b}$ & $2.23 \pm 0.31 \mathrm{~b}$ & $1.90 \pm 0.66 \mathrm{c}$ & $\begin{array}{c}3.60 \\
\pm 0.20 \mathrm{a}\end{array}$ & $2.40 \pm 0.60 \mathrm{~b}$ & $1.33 \pm 0.24 \mathrm{c}$ & $0.83 \pm 0.03 \mathrm{~d}$ \\
\hline $\mathrm{T} 7$ & $\begin{array}{c}1.70 \\
\pm 0.26 \mathrm{c}\end{array}$ & $1.96 \pm 0.13 \mathrm{c}$ & $2.30 \pm 0.3 b$ & $2.10 \pm 0.2 b$ & $\begin{array}{c}3.40 \\
\pm 0.45 \mathrm{a}\end{array}$ & $2.00 \pm 0.2 b$ & $1.27 \pm 0.20 \mathrm{c}$ & $0.90 \pm 0.05 \mathrm{~d}$ \\
\hline $\mathrm{Ta}$ & $\begin{array}{c}1.70 \\
\pm 0.26 \mathrm{c} \\
\end{array}$ & $2.13 \pm 0.26 \mathrm{~b}$ & $2.77 \pm 0.18 b$ & $2.77 \pm 0.43 b$ & $\begin{array}{c}3.52 \\
\pm 0.56 \mathrm{a} \\
\end{array}$ & $2.13 \pm 0.53 b$ & $1.06 \pm 0.16 \mathrm{c}$ & $0.87 \pm 0.06 \mathrm{~d}$ \\
\hline
\end{tabular}

Ta - Termitomyces albuminosus (MTCC 1366). Values are mean \pm SE.

Means in a column followed by a same letter (s) are not significantly $(p \leq 0.05)$ different according to DMRT.

\section{Discussion}

The intriguing activities and properties of termites none seem as widely recognized or as often quoted as their ability to utilize wood as food source. Many species prefer wood that is partially degraded by the associative fungi [28]. Termites especially the sub family macroterminae is known to form symbiosis with Termitomyces. Members of the two major fungus-growing termite genera, Macrotermes and Odontotermes, process plant biomass in a similar way [29] involving two gut passages and external decomposition in fungal gardens. Old workers collect plant substrate and transport the young workers ingest the plant material along with asexual Termitomyces spores produced in fungal nodules in the mature parts of the fungal comb. This mixture passes through young termite gut (first gut passage), which possibly contributes to lignin cleavage [30]. Mushrooms have been reported to produce a wide range of secondary metabolites having high therapeutic values such as antioxidant, antitumor, antibacterial, antiviral, cholesterol lowering, hematological agents and immunomodulating properties [31]. Lignocellulose is a heteropolymer consisting mainly of three components, cellulose, hemicellulose and lignin [32, 
33]. The characteristics of these components are summarized, with the major enzymes responsible for their degradation in the termite mound.

\section{Isolation of Termitomyces spp from Termite Ecosystems}

Termitomyces spp. has become highly valued, partially due to their rareness and difficulty in cultivation [34]. Termitomyces spp. remain elusive and even laboratory trials to culture and cultivate them are far from success stories. Pure cultures of $T$. heimii were obtained using tissue culture and spore inoculation methods. It was easily obtained when young sporophores are used [35]. In the present study, twenty isolates were obtained from the mushrooms and termite mound collected from different locations using tissue culture method generally enriched in Potato Dextrose Agar (PDA) medium. Since Termitomyces is a slow grower in PDA, a selective media namely malt extract medium was used which enhanced the growth of fungus. Malt extract media is recommended for the detection, isolation and enumeration particularly for fungi in various materials and cultivation of this mould for microbiological vitamins and other antimicrobial compounds study. PDA and Malt extract media supported the growth of Termitomyces mycelia than the other culture media [36]. T. umkowaani showed an increased growth rate of up to $18 \mathrm{~mm}$ in Malt extract media in 5 days as compared to $12 \mathrm{~mm}$ in [36].

\section{Morphological and Cultural Characterization of the Isolates}

A detailed classification of Termitomyces based on the macro and micro morphological characters was given by Tibuhwa et al. [17]. Macro morphologically, three groups were classified based on the cap size. Morphological characterization can be done based on shape of perforatorium, stipe length $(\mathrm{cm})$, pileus length, margin of fruit body, colour of fruit body, gills, flesh, annulus, pseudorrhiza and spore print [18] .

In the present investigation, the mushrooms collected from different locations were studied for their cap size, colour of the fruiting body, gills, flesh, etc for the identification of the genus macro morphologically. Based on the observations, the colour of the cap was varying from white cap with brown centre to grey cap with brown centre. Other morphological characters were also studied and the genus of some of the collected mushrooms was found to be Termitomyces. In the present study, the microscopic observation of the isolates revealed that the isolates produced white, turning creamy, soft, hymenophoraltrama regular with thin walled narrow, parallel surface had hyphae forming a hypodermal layer below the epicutis consisting of radially parallel, repent, narrow hyphae.

\section{Enzyme Production by Termitomyces spp.}

Termitomyces clypeatus is known as a potential producer of different enzymes in culture media [37]. Production of lignocellulosic enzymes (cellulase, xylanase, amylase, poly phenol oxidase and peroxidase) by Termitomyces isolates in present study consistent with earlier studies. Different enzymes such as endo1,4-D-Xylanase, 1,4-D-Xylosidase, $\quad \alpha$-Lrabinofuranosidase, acetyl esterase, $\alpha$-amylase, and amyloglucosidase were also purified from T. clypeatus [37]. Ghosh and Sengupta [12] screened the lignocellulolytic enzymes from T. clypeatus and found their commercial utility to produce cellulase, amylase and xylanase under submerged condition in good titre. Ghosh and Sengupta [38] further studied the production of extracellular xylanase and amylase under submerged condition of $T$. clypeatus.

The isolate $\mathrm{T} 1$ recorded maximum cellulose activity in present study accordance with the studies of Zhang et al. [39] who reported the production of lignocellulolytic enzymes e.g., cellulase, xylanase, amylase, laccase and peroxidase production under submerged fermentation of Termitomyces eurrhizus. Termitomyces conidiophores associated with the fungus garden of M. michaelseni produces a cellulase and Termitomyces is a very good source for cellulose system [40]. Gupta et al., 2014 reported that Termitomyces spp. OE147 has been identified as a fungus that fully mineralizes woody material and produces large quantities of $\mathrm{CDH}$ [16] unlike most of the cellulolytic microorganisms. However, Termitomyces spp. isolated from Termite Ancistiermus cervithoras and Microterms toumodiens is have very low enzymatic activities. This was supported by Sawhasan et al. where, Termitomyces clypeatus isolated from the comb of Hypotermyces makhamensis have very low cellulose activities. Production of lignocellulolytic enzymes are mostly influenced by various inorganic additives and temperatures [41, 42]. The mushroom fungus Lentinus conatus elaborated maximum production of endocellulase $(12.75 \mathrm{U} / \mathrm{ml})$ and exocellulase $(8.30 \mathrm{U} / \mathrm{ml})$ in liquid and solid substrates [43].

Xylanase catalyzes the hydrolysis of xylopyranosyl linkages of $\beta 1,4$ xylan, a plant polysaccharide next to cellulose in abundance. Owing to the increasing biotechnological importance of thermostablexylanases, many thermophilic fungi have been examined for xylanase production [44]. All the Termitomyces isolates have xylanase activity in this study is in accordance with the finding of Mukherjee and Sengupta who reported that the mushroom Termitomyces clypeatus produced an endoxylanase $(1,4-$ beta -D-xylanxylanohydrolase) in the presence of either dextrin or xylan as sole source of carbon. Lakshmanan et al. [45] studied the lignocellulolytic enzymes production by Lentinus conatus and Pleurotus sajor-caju with various inorganic additives and the results revealed maximum production by addition of inorganic additives. Gianni et al. [46] observed production of xylanolytic enzymes $(304 \mathrm{U} / \mathrm{g})$ by Fusarium oxysporum grown on corn stover in solid state fermentation. Yang et al. [47] also found the production of extracellular xylanase under optimized 
condition yields $18580 \mathrm{U} / \mathrm{g}$ of xylan. Faulet et al. [48] indicated that thermostable xylanase from Termitomyces exhibited $80 \mathrm{kDa}$ by gel filtration at $65^{\circ} \mathrm{C}-70{ }^{\circ} \mathrm{C}$ and $\mathrm{pH}$ $5.0-6.0$.

Amylase has been derived from several fungi, yeasts, bacteria and actinomycetes, however enzymes from fungi have dominated applications in industrial sectors [49]. Kathiresan and Manivannan [50] reported $\alpha$ - amylase production by Penicillium fellutanum isolated from mangrove rhizosphere soil. Ghosh and Sengupta [36] purified amylase from the culture filtrate of Termitomyces clypeatus by ammonium sulphate precipitation DEAESephadex chromatography and gel filtration. Specific activity of amylase was 3.38 (U/mg protein) in the culture filtrate.

Basidiomycetes, which cause white rot decay, are able to degrade lignin in lignocellulosic wastes. White rot fungi produce extracellular phenoloxidase, which are responsible for initiating the depolymerization of lignin [51]. Maximum laccase activity was exhibited by $P$. djamor in paddy straw substrate, 10 days after inoculation [52]. High enzymes activities of laccase, poly phenol oxidase and peroxidase were observed in Lentinus conatus at $25^{\circ} \mathrm{C}$, compared with Pleurotus sajor-caju [44]. Similar observation was also reported by Ganesh kumar [53] and Thanikaivelan [43].

Banik et al. [54] reported Cellobiase (b-glucosidase) is a well characterized enzyme of the cellulose multienzyme system which acts synergistically to degrade cellulose into constituent glucose subunits, the raw material thiol groups play a pertinent role in modulating the activity of both extracellular and intracellular cellobiase purified from the filamentous fungus Termitomyces clypeatus [54] and inclusion of reducing agents such as dithiothreitol (DTT), stimulated cellobiase activity in the fungus [55] Cellulase, lignin peroxidase and manganese peroxidase was assayed in Aspergillus, Fusarium, Mucor, Rhizopus, Trichoderma and some unidentified genera [56]. Lignin peroxidase activity was done using the method of Berridge [57]. Moreover, in present study all the Termitomyces isolates produced peroxidase. Glucose as carbon source and a mixture of yeast extract and peptone at the ratio of 3 to 5 as nitrogen source in a production medium were shown to produce highest peroxidase activity $(73 \mathrm{U} / \mathrm{ml})$ by Arthromyces ramosus [58].

\section{Conclusions}

Producing fuel ethanol from inedible and abundantly available cellulose biomass offers an important opportunity to sustainably produce alternative transportation fuel. Although significant progress has been made to reduce the manufacturing costs, widespread commercialization of this technology has not been realized. The biofuel industry needs way to combine integrated production technologies and produce value-added byprooducts. A milestone in the scientific development would be microorganisms that can convert lignocellulosic biomass into ethanol efficiently and therefore would enable consolidated bioprocessing to become commercially attractive. Moreover, the development of naturally lingo cellulolytic microorganisms could be resumed, because this might solve the problem as well or could at least improve our understanding cellulosomal enzymes and how they work together. Currently many efforts are devoted to developing new technologies in further decrease the cost of pretreatment and generate less toxic chemicals, higher sugar yield and higher value byproducts through microbial enzymes.

\section{Acknowledgement}

Authors highly acknowledged Department of Science and Technology, New Delhi, for financial assistance (DST/SSTP/TN09/88).

\section{REFERENCES}

[1] Ulrich, G. M. and G. Nicole. 2002. Fungus farming insects: Multiple origins and diverse evolutionary histories, University of Texas, Austin. pp.447.

[2] Heim, R. 1977. Termites et Champignons. Les termitophiles d'Afrique Noire at d'Asie Meridionale. Societe Novelle des Edition, Paris, France. pp 205.

[3] Fang, H.Y., S.M. Chang, M.C. Hsieh and Fang, T.J. 2007. Production, optimization growth conditions and properties of the xylanase from Aspergillus carneus M34. J. Mol. Catal. B: Enzymatic 49: 36-42.

[4] Breznak, J.A. and A, Brune. 1994. Role of microorganisms in the digestion of lignocellulose by termites. Ann. Rev. Entomol. 39:453-487.

[5] Makonde, H. M., H. I. Boga, Z. Osiemo, R. Mwirichia, J. B. Stielow, M. Goker, H.P. Klenk, 2013. Diversity of Termitomyces associated with fungus-farming Termites assessed by cultural and culture-independent methods. PLoS ONE 8 e56464.

[6] Srivastava, B., A.K. Dwivedi, V. N. Pandey, Sociobiology and natural adaptation of Termite and Termitomyces in different forest division of Gorakhpur region. Bull. Env. Pharmacol. Life Sci.2 (2012) 32-36.

[7] Martin, M. M. 1983. Cellulose digestion in insects. Comp. Biochem. Physiol. 75A:313-324

[8] Darlington, J.E.C.P. 1994. Nutrition and evolution in fungus-growing termites. In: Hunt JH, NalepaCA(eds) Nourishment and evolution in insect societies. Westview Press, Boulder, CO, pp 105-130

[9] Hyodo, F., T. Inoue., J. I. Azuma., I. Tayasu and T. Abe. 2000. Role of the mutualistic fungus in lignin degradation 
in the fungus-growing termite Macrotermes gilvus (Isoptera; Macrotermitinae). Soil Biol. Biochem. 32: 653-658.

[10] Johjima, T., M. Ohkuma and T. Kudo. 2003. Isolation and cDNA cloning of novel hydrogen peroxide-dependent phenol oxidase from the basidiomycete Termitomyces albuminosus. Appl. Microbiol. Biotechnol. 61: 220-225.

[11] Taprab, Y., T. Johjima., Y, Maeda., S. Moriya., S. Trakulnaleamsai., N. Noparatnaraporn., M. Ohkuma and T. Kudo. 2005. Symbiotic fungi produce laccases potentially involved in phenol degradation in fungus combs of fungus-growing termites in Thailand. Appl. Environ. Microbiol. 71: 7696-7704.

[12] Ghosh, A.K. and S. Sengupta. 1982. Production of extracellular carbohydrases by mushrooms. Acta Mycol. 18: 113.

[13] Kendrick B 2001. The Fifth Kingdom ( $3^{\text {rd }}$ ed.). Sidney, BC, Mycologue Publisher, Canada.

[14] Johjima, T., M. Ohkuma and T. Kudo. 2003. Isolation and cDNA cloning of novel hydrogen peroxide-dependent phenol oxidase from the basidiomycete Termitomyces albuminosus. Appl. Microbiol. Biotechnol. 61: 220-225.

[15] Chang, S.T, 1982. Cultivation of Volvariella mushrooms in Southeast Asia. In: S.T. Chang, and T.H. Quimio (Eds.), Tropical mushrooms: Biological nature and cultivation methods. The Chinese Univ. Press, Hong Kong.

[16] Rangasamy, G., T.K. Kandasamy and K. Ramasamy. 1975. Pleurotus sajor-caju (Fr.) Singer - protein rich nitrogen fixing mushroom fungus. Curr. Sci., 44: 403-444.

[17] Tibuhwa, D.D., A. K. Kivaisi and F. S. S. Magingo. 2010. Utility of the macro-micromorphological characteristics used in classifying the species of Termitomyces. Tanz. J. Sci. 36: 31-45.

[18] Srivastava, B., A. K. Dwivedi and V. N. Pandey. 2011. Morphological characterization and Yield Potential of Termitomyces spp. Mushroom in Gorakhpur forest Division. Bull. Environ. Pharmacol. Life Sci. 1: 54-56.

[19] Tibuhwa, D. D. 2012. Termitomyces species from Tanzania, their cultural properties and unequalled Basidiospores. J. Biol. Life Sci. 3: 1-20.

[20] Kawato, M, and R. Shinobu. 1979. A simple technique for the microscopical observation. Memoirs of the Osaka University Liberal Arts and Education. 114p.

[21] Miller, G.L. 1959. Use of dinitrosalicylic acid reagent for determination of reducing sugar. Anal. Chem. 31: 426-428.

[22] Chen, W.P., M. Matsuo and T Yasui. 1986. Purification and properties of

3-1,3-xylanase from Aspergillus terreus A-07. Agricul. Biol. Chem. 50: 1183-1194.

[23] Nelson, N. 1944. A photometric adaptation of the Somogyi methods for the determination of glucose. J. Biol. Chem. 153: 375-377.

[24] Somogyi , N. 1952. A photometric adaptation of the Somogyi method for the determination of glucose. J. Biol. Chem. 153: 375-380.

[25] Ghosh, A. K., C. Banerjee and S. Sengupta. 1980.
Purification and properties of xylan hydrolase from mushroom Termitomyces clypeatus. Biochem. Biophys. Acta. 612: 143-152.

[26] Zuber, M., and K. Manibhushanrao. 1982. Studies on comparative gel electrophoretic pattern of protein and enzymes from isolates of Rhizotonia solani causing sheath blight disease in rice. Can. J. Microbiol. 28: 762-771.

[27] Putter, J. 1974. Peroxidase. In: Methods of Enzymatic Analysis. H.U. Bergemeyer (Ed.). Vol. II. Academic Press, London. pp. 685-690.

[28] Ajit, V., K. Ballakrishnan, P. Jaishree, S. Shailendra and K. Helmut. 1994. Lignocellulose degradation by microorganisms from termite hills and termite guts: A survey on the present state of art. FEMS Microbiol. Rev. 15: $9-28$.

[29] Li, H., M. Yang, Y. Chen, N. Zhu, C.Y. Lee, J.Q. Wei, J. Mo. 2015. Investigation of age polyethism in food processing of the fungus-growing termite Odontotermes formosanus (Blattodea: Termitidae) using a laboratory artificial rearing system. J Econ Entomol 108:266 -273.

[30] Li H, Yelle DJ, Li C, Yang M, Ke J, Zhang R, Liu Y, Zhu N, Liang S, Mo X, Ralph J, Currie CR, Mo J. 2017. Lignocellulose pretreatment in funguscultivating termite. Proc Natl Acad Sci U S A 114:4709-4714

[31] Yang, J.H., H.C. Lin and J.L. Mau. 2002. Antioxidant properties of several commercial mushrooms. Food Chem. 77: 229-235.

[32] Fengel, D. and G. Wegener. 1989. In: Wood: Chemistry, Ultra structure Reactions. New York, USA.

[33] Eaton, R.A. and M. D. C. Hale. 1993. In: Wood, Decay, Pests and Prevention. Chapman and Hall, London, UK.

[34] Tsai, S.H., T.P. Wu, S.J. Huang and J.L. Mau. 2006. Nonvolatile taste components of Agaricus bisporus harvested at different stages of maturity. Food Chem. 103: 1457-1464.

[35] Muid. S. 1992. In: Isolation and growth of Termitomyces heimii cultures on some artificial media. Proc. Asian Mycol. Symp., pp. 286-291.

[36] Ghosh, S., N. Paweletz and I. Ghosh. 1978. Mitotic asynchrony of multinucleate cells in tissue culture. Chromosoma 65: 293-300.

[37] Khowala, S. and S. Sengupta. 1992. Secretion of $\beta$-glucosidase by Termitomyces clypeatus. Enzyme Microb. Tech. 14: 144-149.

[38] Ghosh, A.K. and S. Sengupta. 1987. Multisubstrate specific amylase from mushroom Termitomyces clypeatus. J. Biol. Sci. 11:275-285.

[39] Zhang, M., Z. Xiao-dong, C. Xin-ai and Y. Yuan-ming. 2002. Study on the submerged fermentation of Termitomyces eurrhizus. J. Zhejiang Univ. Sci. 3: 362-366.

[40] Abo-khatwa, A.N. 1989. Termitomyces: A new source of potent cellulases. J. K. A. U. Sci. 1: 51-59.

[41] Ardon, O., Z. Kerem and Y. Hardar. 1996. Enhancement of laccase activity in liquid cultures of the lignolytic fungus Pleurotus ostreatus by cotton stalk extract. J. Biotechnol. 


$$
\text { 51: 201-207. }
$$

[42] Bommaraju, P. 2002. Studies on lignocellulolytic break down of certain agro-industrial wastes by Lentinus connatus Berk. for production of potential biomanure. (Unpublished Thesis), Tamil Nadu Agricultural University, Coimbatore, India.

[43] Thanikaivelan, K. 2003. Production of extracellular lignolytic enzymes by Lentinus connatus. (Unpublished Thesis), Konganadu Arts and Science College, Coimbatore, India.

[44] Singh, R., D.N. Kamra, K. Neelam and P. Rakesh. 2003. Production of extracellular enzymes by certain lignocellulose degrading white rot fungus. Int. J. Animal. Sci. 9: 105-107.

[45] Lakshmanan, P., R. Jagadeesan, A. Sudha, M. Rajesh, S. Prabhakara and P. M. Arun. 2008. Potentiality of a new mushroom fungus Lentinus connatus Berk for the production of biomanure from sugarcane trash (Saccharum officinarum L.) and its impact on the management of groundnut root rot diseases. Arch. Phytopathol. Plant Protect. 41: 273-289.

[46] Gianni, P., K. Dimitris, B. J. Macris, and P. Christakopoulos. 2007. Production of cellulolytic and xylanolytic enzymes by Fusarium oxysporum grown on corn stover in solid state fermentation. Ind. Crop. Prod. 18: 37-45.

[47] Yang, S. Q., Q. J. Yan, Z. Q. Jiang, L. T. Li, H. M. Tian and Y.Z. Wang. 2006. High-level of xylanase production by the thermophilic Paecilomyces themophila J18 on wheat straw in soild- state fermentation. Bioresour. Technol. 97: 1794-1800.

[48] Faulet, B.M., S. Niamke, J.T. Gonnety and L.P. Kouame. 2006. Purification and biochemical properties of a new thermostable xylanase from symbiotic fungus, Termitomyces sp. Afr. J. Biotechnol. 5: 273-282.

[49] Pandey, A. 2002. Recent process developments in solid state fermentation. Process Biochem. 27: 109-117.
[50] Kathiresan. K and S. Manivannan. 2006. $\alpha$-Amylase production by Penicillium fellutanum isolated from mangrove rhizosphere soil. Afr. J. Biotechnol. 5: 829-832.

[51] Ohkuma, M. Y., T. Maeda, T. Tohjima and K. Toshiaki. 2001. Lignin degradation and role of white rot fungi: study on an efficient symbiotic system in fungus-growing termite and its application to bioremediation. Annu. Rev. Microbiol. 41: 465-472.

[52] Ravichandran. V. 2001. Studies on developing high yielding Oyster mushrooms with desirable qualities. (Unpublished Thesis), Tamil Nadu Agricultural University. Coimbatore, India.

[53] Ganeshkumar, V. 2003. Enzymes production potential of Pleurotus djamor. (Unpublished Thesis). Kongunadu Arts and Science College, Coimbatore, India.

[54] Banik S.P., S. Pal, S. Ghorai, S. Chowdhury, R. Majumder, S. Mukherjee, S. Khowala. 2012. In situ reversible aggregation of extracellular cellobiase in the flamentous fungus Termitomyces clypeatus. Biotechnol Bioprocess Eng 17: 925-936

[55] [55] Pal, S., S.P. Banik, S. Ghorai, S. Chowdhury, S. Khowala. 2010. Purification and characterization of a thermostable intracellular b-glucosidase with transglycosylation properties from filamentous fungus Termitomyces clypeatus. Biores Technol 101:2412-2420

[56] [56] Afolabi, O.O., O. E. Fagade and A. A. Ogunjobi. 2010. Enzymatic activity of some fungal isolates from decaying bamboo leaves. J. Life Phy. Sci. 3: 108-112.

[57] [57] Berridge, N.J. 1955. Methods in Enzymology .Academic Press. pp185-192.

[58] [58] Tsujimura. H., M. Takaya, K. Katano, N. Matsumoto, Y. S. Park and M. Okabe. 1994. Peroxidase production by carbon and nitrogen sources fed-batch culture of Arthromyces ramosus. Biotechnol. Lett. 16:575-580. 